\title{
Janis Ehling
}

\section{Die deutsche Sozialdemokratie vor dem Untergang - zerrieben zwischen Kosmopolitismus und Kommunitarismus?}

„Social Democracy's at Death's Door“, „Der Untergang der Sozialdemokratie ist nicht aufzuhalten" so und ähnlich lauten die Untergangsszenarien. ${ }^{1}$ In den letzten Jahren erhalten sie zusätzliche Brisanz, weil die Verluste der Sozialdemokratie nicht mehr die Gewinne anderer Linksparteien sind. Als politische Alternativen zum sozialdemokratischen Wohlfahrtsstaat stehen ein liberal-konservativer Kapitalismus à la Schwarz-Grün und ein autoritärer Kapitalismus bereits in den Startlöchern (Deppe 2013). Vor diesem Hintergrund erscheint manchen die Krise der Sozialdemokratie als Existenzkrise der Linken insgesamt. So verstanden, dauert die Existenzkrise der Sozialdemokratie schon seit vier Jahrzehnten an (vgl. Walter/Micus 2011: 383ff.). Hier wird jedoch - vorsichtig formuliert - der Krisenbegriff arg überstrapaziert.

Die Strukturprobleme der Sozialdemokratie rücken wieder in den Blickpunkt, weil die derzeitige Formierung der Rechten zunehmend Teile der Arbeiterklasse umfasst. Die Entwicklung der Sozialdemokratie ist nicht neu, aber ihre Bedeutung für die Gesellschaft als Ganzes führt dazu, dass sie von der Öffentlichkeit derzeit neu entdeckt wird. Auch durch das Buch

1 „Social democracy at Death's door“ (Balhorn 2019); „SPD: Warum der Untergang der SPD nicht aufzuhalten ist (Rehage 2018).
Rückkehr nach Reims von Didier Eribon wird die Zukunft der politischen Linken als Klassenfrage mitverhandelt. Die Pole der Diskussion reichen vom Plädoyer für die Aufgabe einer Klassenpolitik (Seibert 2018) und der Hinwendung zu „progressiven politischen Koalitionen jenseits der Lohnabhängigenbasis“ (Kitschelt 2013: 225) bis hin zum Rückbezug auf die soziale Frage im Umfeld der SPD bei Wolfgang Merkel, Nils Heisterhagen und Andreas Nölke.

Die derzeitige Diskussion bleibt jedoch oberflächlich, denn die Entwicklung der Klassen hat sich in der sozialdemokratischen Organisationsentwicklung spezifisch ausgedrückt. Die bisherigen Vorschläge zur Lösung der Strukturprobleme der Sozialdemokratie berücksichtigen das zu wenig.

\section{Krise der Sozialdemokratie - Auflösung der Klassengegensätze?}

Die derzeit diskutierte Krise der Sozialdemokratie besteht aus (lösbaren) Strukturproblemen des derzeitigen sozialdemokratischen Parteityps. Die (west-)europäischen Parteiensysteme sind seit drei Jahrzehnten stark in Bewegung. Dem aktuell beklagten Untergang der Sozialdemokratie ging eine rasante Transformation von Parteien und gesellschaftlichen Gruppen vorweg. Parteien entstehen aus gesellschaftlichen 
cleavages (Konfliktlinien) (kritisch: PROKLA Redaktion 2018). Eine Partei - im gramscianischen Sinne als gesellschaftliche Gruppe - verschwindet erst, wenn der Konflikt, aus dem die Partei entstanden ist, gelöst ist. Entsprechend reichen die Organisationen und Traditionen der deutschen Parteien 150 Jahre zurück - eine bemerkenswerte Konstanz. Die zentralen gesellschaftlichen Konfliktlinien haben sich nicht wesentlich verändert.

Die cleavage, die der Sozialdemokratie ihre Existenz verdankt, ist der Konflikt zwischen Kapital und Arbeit, der class cleavage. Er ist im Vergleich der letzten 150 Jahre einer der stabilsten cleavages überhaupt, wenn er sich in den letzten Jahrzehnten auch abgeschwächt hat (vgl. Bartolini/Mair 1990: 77ff.). Für die deutsche Soziologie ist der Befund hingegen klar: Stellvertretend bedient Andreas Reckwitz in Die Gesellschaft der Singularitäten den Evergreen der deutschen Mainstreamsoziologie, die Individualisierung sei ursächlich für den Niedergang der Sozialdemokratie (vgl. Raphael 2019: 109ff.). Die Kategorie Klasse muss in Deutschland zur Selbstvergewisserung einmal pro Jahrzehnt öffentlichkeitswirksam wissenschaftlich beerdigt werden: ob bei Schelsky, Beck oder nun in Andreas Reckwitz' Die Gesellschaft der Singularitäten. Dabei ist die These damals wie heute falsch (vgl. Ritsert 1998: 88ff.; Vester 2019: 40ff.). Das gilt auch für die Parteienforschung. Alle Vorhersagen nach Gründung der Partei Die Grünen die Postmaterialismusdiskussion wie die dealignment-Theorie - haben sich so nicht bestätigt (vgl. von Beyme 2000a: 66). Die Grünen haben wie die neuen rechten Parteien eine Klassenbasis (vgl. Oesch 2013: 31ff.; Sablowski/Thien 2018: 58ff.).

\section{Krise der Parteien als Krise der Linken}

Die allgemeine Krise der Parteien ist hingegen vor allem eine Krise der Linken. Die Mitglieder der SPD haben sich seit 1990 nahezu halbiert. Damit reiht sie sich in die allgemeine Entwicklung der sozialdemokratischen Parteienfamilie ein (vgl. Jun 2012: 86ff.). Das könnte man mit Verweis auf die CDU und ihre fast ebenso hohen Verluste kleinreden. Doch die parteipolitische Linke ist ungleich stärker von der Krise der Parteien betroffen (Mair 2003: 6ff.). Der Grund ist einfach. Wer etwas verändern will, aber nicht Teil der herrschenden Klasse ist, muss Massen mobilisieren und braucht starke Organisationen als Machtressourcen. Zur Konfliktaushandlung innerhalb der Elite reichen in einer liberalen Demokratie kleine Wahlvereine völlig aus. Die ersten Parteien im 19. Jahrhundert waren entsprechend Parlamentsclubs und lokale Honoratiorenvereinigungen. ${ }^{2}$

Erst mit den linken Massenparteien sahen sich die Konservativen und Rechten veranlasst, ebenfalls auf Massenorganisationen zu setzen. Mit dem modernen Parteiensystem entstand nach dem Zweiten Weltkrieg in Deutschland das, was Claus Offe als die Herausbildung des „Formprinzips der Konkurrenzpartei" beschrieben hat, mit der auch eine Logik der Entpolitisierung der cleavages einhergeht: „Die Logik der nach ,Regierungsverantwortung' strebenden Konkurrenzpartei legt es den Parteien nahe, Wählerstimmen zu suchen, wo immer sie zu bekommen sind, sich also jeder Bezugnahme auf (klassenmäßige, konfessionell

2 Und auch heute geht der Trend auf der lokalen Ebene weg von Massenparteien wie der CDU zu kleineren konservativen Honoratiorenvereinen. 
oder sonst wie) spezialisierte ,Einzugsbereiche' zu enthalten. Dementsprechend wird der Bürger als abstraktes Willenssubjekt, als ein mit Stimmrecht ausgestatteter Jedermann angesprochen." (Offe 1980: 31) Dieses Formprinzip ist auch in der gegenwärtigen Strategie der Konservativen zu finden, nicht erst seit Angela Merkel, als Strategie der Entpolitisierung, die die Forschungsgruppe Wahlen 2009 als ,asymmetrische Demobilisierung" der WählerInnen bezeichnet (Forschungsgruppe Wahlen 2009). Schon Merkels politischer Ziehvater Helmut Kohl hatte sich dieser Strategie bedient, wie Joachim Hirsch und Josef Esser in der PROKLA 56 zum „CDU-Staat" herausarbeiteten: „Die entscheidende Rolle der Partei besteht in der Organisierung eines politisch-ideologischen Diskurses [...] Dieser Diskurs ist entpolitisierend in dem Sinne als er systematisch Themen und Konflikte ausklammert, die zu einer Re-Formierung übergreifender Klassen- und sozialer Konfliktfronten führen könnte" (Hirsch/Esser 1984: 61). Diese gezielte Demobilisierung des Klassenkonflikts wurde von der SPD durchaus mitbefeuert - bereits wenige Jahre nach Gründung der Bundesrepublik.

\section{Krise des sozialdemokratischen Parteimodells in Deutschland}

Auf dem Bad Godesberger Parteitag 1959 wählte die deutsche Sozialdemokratie die Transformation der eigenen Partei von einer ArbeiterInnen- zu einer Volkspartei. Aus Machtkalkül war das sinnvoll. Die Basis der SPD, die IndustriearbeiterInnen, machten selbst zu Hochzeiten nicht mehr als 40 Prozent der Bevölkerung aus. Daher wollte die SPD weitere Klassenfraktionen erreichen. Diese „catch-all-Party“ (Kirchheimer 1965) expandierte schnell in andere Milieus. Die Zahl der ArbeiterInnen in der SPD ging hingegen im Verhältnis zu anderen Gruppen zurück - angefangen bei den Führungsspitzen bis hin zur Parteibasis. Lag der ArbeiterInnenanteil der SPD-Bundestagsfraktion 1956 noch bei 44 Prozent, waren es 1976 nur noch 2,2 Prozent. Ab 1974 waren ArbeiterInnen innerhalb der SPD erstmals nicht mehr in der Mehrheit. Seit den 1970ern akademisierte sich die SPD zunehmend. Ab den Unterbezirken der Parteiorganisation dominieren seit den 1980ern BerufspolitikerInnen mit akademischem Abschluss (vgl. Lösche/ Walter 1992: 131ff.). Dabei legen von allen Gruppen die ArbeiterInnen den höchsten Wert auf die soziale Repräsentation ihrer Klasse (vgl. von Beyme 2000b: 334). Die Sozialdemokratie hatte sich in eine professionalisierte Medienpartei verwandelt, die den Kontakt zu ihren Mitgliedern vernachlässigt und mehr auf Medienrepräsentanz setzt - die parteieigenen Medien wurden überwiegend eingestellt (vgl. von Beyme 2000a, 2000b).

Die eigentliche Mitgliederarbeit spielte dahingehend kaum noch eine Rolle. Dabei sind die klassischen Arbeitermilieus deutlich weniger an Karrieren interessiert, sondern aus ideologischen Gründen Mitglied einer Partei oder auf der Suche nach Geselligkeit und Veranstaltungen. Mitglieder mit geringen Bildungsabschlüssen sind dabei deutlich aktiver in Parteien als Hochschulabsolventen (vgl. Spier 2011: 100ff.). Die Entkopplung von Partei und Gewerkschaften tut ihr übriges: der Anteil an ArbeiterInnen lag in der SPD schon 2010 bei nur noch 11 Prozent, der Anteil an GewerkschafterInnen 2018 bei nur noch 34 Prozent. Die Zahl der Gewerkschaftsmitglieder unter den SPD-Mitgliedern sinkt weit schneller als in der Gesamtbevölkerung (vgl. Walter 2010: 118f.; Klein u.a. 2019). Die SPD ist heute mehr eine Partei der Angestellten, RentnerInnen und des Öffentlichen Dienstes als eine 
ArbeiterInnenpartei. Die alten StammwählerInnen und-Mitglieder der SPD sind überwiegend ins Nichtwählerlager abgewandert. Von 400.000 verlorenen Mitgliedern der SPD seit 1990 sind kaum 2.000 in die WASG (Arbeit \& soziale Gerechtigkeit - Die Wahlalternative) gegangen, die sich 2004 vor allem aus regierungskritischen SPD-Mitgliedern gründete (und $2007 \mathrm{der}$ PDS zur Partei DIE LINKE fusionierte). Deutlicher formuliert: Die Sozialdemokratie lebt schon länger von der Substanz ihrer älteren und vor Jahrzehnten rekrutierten Mitgliedschaft.

Parallel zu diesen gravierenden organisatorischen Entwicklungen kommen die strukturellen Entwicklungen, die von u.a. Wolfgang Merkel, Andreas Nölke, Thomas Seibert zu wenig beachtet werden: Seit den 1980er Jahren ging eine Deindustrialisierung einher mit einem Wandel der ArbeiterInnenklasse, der Schwächung der Gewerkschaften als wichtige sozialdemokratische Machtressource, dem Wegzug der IndustriearbeiterInnen aus den Städten und dem Verfall der sozialdemokratischen Milieus. Die vormaligen Hochburgen der Mittel- und Großstädte sind eher der Ort Dienstleistungsklassen als Industriestandorte. Hier dominieren die classes populaire. ${ }^{3}$ Sie sind aber weit weniger politisch und gewerkschaftlich organisiert und nehmen sich weniger als gesellschaftliche Kraft war (Raphael 2019: 138ff.). Das Verschwinden der (Industrie-)ArbeiterInnen aus der Politik, der Basis der SPD, hat sie durch ihre Orientierung auf die „Neue Mitte“ weiter

3 Der Begriff ist ein Notbehelf, denn trotz noch bestehender Unterschiede haben sich die Lebensverhältnisse von ArbeiterInnen und Angestellten angenähert. Der Begriff ist weitaus geeigneter als gängige Begriffe aus der Ungleichheitsforschung (vgl. Raphael 2019: 138ff.; Thien 2018: 213f.). beschleunigt (Nachtwey 2009: 173ff.). Heute setzt sich die SPD zusammen aus ArbeiterInnen, AkademikerInnen, Beschäftigte im Öffentlichen Dienst und vor allem RentnerInnen (vgl. Walter 2010: 117ff.). ${ }^{4}$ Mit den Grünen und der Linkspartei gibt es mittlerweile Politikangebote für die moderne Dienstleistungsklassen im oberen und unteren Segment. Auf die SPD wirken damit gewaltige Fliehkräfte, die sich durchaus auch kulturell reflektieren. Die Antwort der SPD auf diese strukturellen Umbrüche war eine weitere Bewegung in die Mitte. Die Auflösung weiter Teile der Sozialdemokratie in eine übergreifende Mitte-Partei ist eine realistische Option wie die Beispiele in Italien und Frankreich unter Renzi und Macron zeigen. Der Vorschlag des renommierten Parteienforschers Herbert Kitschelt (2013: 225) nach progressiven Koalitionen jenseits der ArbeiterInnenklasse geht in diese Richtung. Für Länder mit tiefer und langer sozialdemokratischer Verankerung ist das keine (vorerst) keine Option. ${ }^{5}$ Europaweit haben die sozialdemokratischen Parteien

4 Die genaue lebensweltliche Untersuchung postindustrieller sozialdemokratischer Milieus ist wenig erforscht. Genauso lässt die Debatte um AkademikerInnen vs. ArbeiterInnen eine Untersuchung der Fachhochschulen vermissen. Fachhochschulstudierende machen mehr als die Hälfte aller Studierenden aus. Deren Universitäten und Universitätslaufbahnen unterscheiden sich jedoch grundlegend von einem Hochschulstudium. Gerade an Fachhochschulen absolvieren die vormaligen und jetzigen Facharbeitermilieus ihre Ausbildung.

5 Es gibt einen großen Unterschied zwischen Ländern mit starker sozialdemokratischer Tradition wie Skandinavien, Österreich, Deutschland, Belgien, Großbritannien und Ländern mit historisch starken kommunistischen Traditionen wie in Frankreich und Südeuropa, wo die Sozialdemokratie immer deutlich schwächer war. Die Niederlande 
in den 1990er Jahren durch die taktische oder tatsächliche Übernahme neoliberaler Politiken zwar große Wahlerfolge eingefahren, diese aber mit der Abspaltung von großen Teilen ihrer Mitglieder und WählerInnen bezahlt (Rhodes 2015: 146ff.). Über die „richtige Machttaktik“ ist der Sozialdemokratie die Strategie abhandengekommen. Die Mitte-Orientierung ist wie das Modell der professionalisierten Medienpartei eine Sackgasse - zerrieben zwischen weiteren Mitgliederverlusten, den medial professionelleren Grünen und der Konkurrenz von links.

\section{Neue Cleavages?}

Eine einseitige Auflösung der beschriebenen Konflikte ist Russisch Roulette. Die Aufgabe ihrer angestammten Klassen- und Klassenfraktionen wie Thomas Seibert (2018) sie vorschlägt, wäre für die Sozialdemokratie politischer Selbstmord. Ohne den class cleavage zu bearbeiten, macht sich die Sozialdemokratie überflüssig. Angesichts der Fragmentierung sozialdemokratischer Klassenfraktionen ergibt der Vorschlag von Wolfgang Merkel durchaus Sinn, sich erstmal auf die sicheren 20 Prozent $+\mathrm{X}$ der WählerInnen zu fokussieren (vgl. Merkel 2018). In einem Sechs- oder Mehrparteiensystem ist eine 20-Prozent+X-Partei ein großer und ernstzunehmender Faktor.

Vor diesem Hintergrund ist zu verstehen, warum Wolfgang Merkel, Nils Heisterhagen und Andreas Nölke dafür eintreten, dass die Sozialdemokratie um „die Unterprivilegierten“ werben müsse. Statt aber ganz auf den class cleavage zu setzen, rufen sie einen neuen cleavage zwischen Kosmopolitismus und Kommunitarismus

sind ein Sonderfall. Daher lassen sich diese Länder schlecht vergleichen. aus. Der politökonomische Strategiewechsel soll bei ihnen ganz auf „kommunitaristische Milieus“ der Unterprivilegierten und weniger auf „kosmopolitische Milieus“ zugeschnitten sein. Die kommunitaristischen Milieus seien eher autoritär, migrationsskeptisch und gegen Identitätspolitik. Eine „härtere“ Gangart in der Migrationspolitik und weniger Identitätspolitik soll die Repräsentationslücke der Partei in diesem Punkt schließen (Merkel 2018; Nölke 2017; Stegemann 2018). Analytisch ist diese Repräsentationslücke zwar bei den verlorenen StammwählerInnen der SPD zu verorten (vgl. Gagné/Hilmer 2019: 49f.). Merkel \& Co. übersehen bei ihrer Kritik an Identitätspolitik zudem leicht, dass die historische SPD natürlich mit dem Fokus auf den männlichen Industriearbeiter hochgradig identitätspolitisch war. ${ }^{6}$ Das augenblickliche Problem ist vielmehr, dass es kaum ein vereinendes Identitätsangebot für die classes populaires gibt. Das zeigen hilflose Begriffe wie „Arme“ und „Unterprivilegierte“ als Bezugspunkte bei Merkel und Nölke. Statt sich Gedanken über eine neue Klassenpolitik zu machen, werden unterschiedliche Aspekte der Lebensführung als Identitätsmarker aufgeblasen (vgl. Raphael 2019: 117). Die Migrationspolitik erscheint Merkel \& Co. so als Hauptunterscheidungsfeld der politischen Linken. ${ }^{7}$

Die Rechte von MigrantInnen hat die SPD in den 30 Jahren bereits sehr weitreichend (mit-)beschnitten. Vergleicht man

6 Ihre Kritik an klassenblinden Linken und progressiven Neoliberalen, die unter RotGrün Frauen rechtlich besser stellten, aber gleichzeitig die soziale Lage von Millionen Frauen verschlechterten, ist völlig richtig.

7 Der erste Sammelband aus dem Forschungsprojekt Die politische Soziologie des Kosmopolitismus und Kommunitarismus heißt bezeichnend The Struggle Over Borders. Cosmopolitanism and Communitarianism. 
unterschiedliche Ansätze sozialdemokratischer Migrationspolitik europaweit ist es für die Wahlergebnisse aber völlig egal wie hart oder weich die Migrationspolitik ist (vgl. Arzheimer 2013: 86ff.). Bestimmt das Thema Migration die Wahlen, nützt das den Rechten, nicht den Sozialdemokraten. Zudem sieht ein größerer Teil der sozialdemokratischen Mitglieder Migration eher als Bereicherung (vgl. Gagné/Hilmer 2019: 25). Die Frage stärkt nicht nur die Rechte, sondern hat einiges Spaltungspotenzial in die SPD-Mitgliedschaft.

Beispiele der sozialdemokratischen Parteien in Portugal, Großbritannien und der Schweiz zeigen hingegen, dass eine Erneuerung ihres polit-ökonomischen Profils durchaus Erfolge zeitigen kann. Auch hierzulande ist politökonomische Repräsentationslücke sowohl bei den WählerInnen als auch den verlorenen Mitgliedern der SPD gerade in Deutschland besonders groß (vgl. Gagné/Hilmer 2019: 16). Die klassenpolitische Revitalisierung der SPD ist möglich.

\section{Fazit}

Ein bloßer Wechsel im Botschaftenmanagement der Sozialdemokratie kann die organisatorisch-kulturelle Vernachlässigung der Industriearbeiterschaft und der classes populaires nicht kompensieren. Der Anspruch der SPD als Volkspartei - hier hat Wolfgang Merkel Recht - lässt sich realistisch nicht aufrecht erhalten. Die Logik der Konkurrenzparteien zwischen zwei großen Parteien wird schwächer und damit auch die Kohäsionskräfte innerhalb der politischen Lager. Die ominöse MitteOrientierung der Sozialdemokratie und die Aufgabe jeder Klassenpolitik mag für einige Zeit elektoral erfolgreich gewesen sein, langfristig hat sie großen Flurschaden angerichtet. In einem Vielparteiensystem werden die Kernklientel wieder wichtiger.
Ohne (Wieder-)Verankerung im Wohnund Arbeitsalltag, ohne eine Repolitisierung der klassischen ArbeiterInnenklasse und der neuen classes populaires wird die politische Linke weiter schrumpfen. Neue cleavages innerhalb der politischen Linken auszurufen, vertieft jedoch eher die Probleme anstatt sie zu lösen.

Die Linke in den USA und die Sozialdemokratie in Großbritannien bringen derzeit die unterschiedlichen politischen Fragen von Klasse, race, Gender und Ökologie unter dem Stichwort Sozialismus zusammen. Die gespaltenen linken Traditionslinien hierzulande machen eine arbeitsteilige Ansprache der Industriearbeiterschaft, der classes populaires und der Unterprivilegierten möglich.

Es geht augenblicklich nicht um den Untergang der SPD, sondern um eine Neudefinition ihrer Rolle im linken Parteienlager.

\section{Literatur}

Arzheimer, Kai (2013): Working-class parties 2.0 ? Competition between centre left and extreme right parties. In: Rydgren, Jens (Hg.): Class politics and the radical right. London: 75-90.

Balhorn, Loren (2019): Social Democracy at Death's Door. In: Jacobine. URL: https:// jacobinmag.com/2019/06/german-socialdemocrats-european-elections, Zugriff: 2.6.2019.

Bartolini, Stefano (2000): Political Mobilization of the left 1860-1980. The class cleavage. Cambridge. https://doi.org/10.1017/ CBO9780511521560

Ders./Mair, Peter (2007): Identity, competition and electoral availability. The stabilization of European electorates 1885-1985. Colchester.

Deppe, Frank (2013): Autoritärer Kapitalismus. Demokratie auf dem Prüfstand. Hamburg.

Forschungsgruppe Wahlen (2009): Bundestagswahl 2009. Eine Analyse der Wabl von 27. September 2009. Mannheim. 
Gagné, Jérémie/Hilmer, Richard (2019): Wo genau ist Mitte-Links? Wert- und PolicyProfile sozialdemokratischer Parteien in Europa - aus Sicht der nationalen Wahlbevölkerungen (value gaps/policy gaps). URL: https://www.fes.de/internationalepolitikanalyse/artikelseite-ipa/wo-genauist-mitte-links, Zugriff: 1.7.2019.

Hirsch, Joachim/Esser, Josef(1984): Der CDUStaat: ein politisches Regierungsmodell für den nachfordistischen Kapitalismus. In: PROKLA 14(56): 51-66. https://doi. org/10.32387/prokla.v14i56.1438

Jun, Uwe (2012): Die sozialdemokratische Parteienfamilie. In: Jun, Uwe/Höhne, Benjamin (Hg.): Parteienfamilien. Identitätsbestimmend oder Etikett? Opladen: 36-51.

Kirchheimer, Otto (1965): Der Weg zur Allerweltspartei. In: Lenk, Kurt/Neumann, Franz (1968): Theorie und Soziologie der politischen Parteien. Neuwied: 345-367.

Kitschelt, Herbert (2013): Social class and the radical right. Conceptualizing party preference formation and partisan choice. In: Rydgren, Jens (Hg): Class politics and the radical right. London: 224-251.

Klein, Markus u.a. (2019): Die Sozialstruktur der deutschen Parteimitgliedschaften. Empirische Befunde der Deutschen Parteimitgliederstudien 1998, 2009 und 2017. In: Zeitschrift für Parlamentsfragen 50(1): 81-98. https://doi. org/10.5771/0340-1758-2019-1-81

Lösche, Peter/Walter, Franz (1992): Die SPD. Klassenpartei-Volkspartei-Quotenpartei. Zur Entwicklung der Sozialdemokratie von Weimar bis zur deutschen Wiedervereinigung. Darmstadt.

Mair, Peter (2003): Political Parties and democracy: What sort of future? In: Central European Political Review (4): 6-20.

Merkel, Wolfgang (2018): SPD muss Anspruch der Volkspartei aufgeben. URL: https:// www.tagesspiegel.de/politik/wolfgangmerkel-spd-muss-anspruch-der-volkspartei-aufgeben/22734200.html, Zugriff: 2.7.2019.

Ders. u.a. (2019): The Struggle Over Borders. Cosmopolitanism and Communtarianism. Cambridge.
Nachtwey, Oliver (2009): Marktsozialdemokratie. Die Transformation von SPD und Labour Party. Wiesbaden. https://doi. org/10.1007/978-3-531-91616-3

Nölke, Andreas (2017): Linkspopulär. Vorwärts handeln statt rückwärts denken. Frankfurt/M.

Oesch, Daniel (2013): The class basis between the new left and the radical right: an analysis for Austria, Denmark, Norway and Switzerland. In: Rydgren, Jens (Hg.): Class politics and the radical right. London: 31-52.

Offe, Claus (1980): Konkurrenzpartei und kollektive politische Identität. In: Roth, Roland (Hg.): Parlamentarisches Ritual und politische Alternativen. Frankfurt/M.-New York: 26-42.

PROKLA Redaktion (2018): Editorial: Autoritärer Populismus Strategie und politische Ökonomie rechter Politik. In: PROKLA. 48(190): 2-8. https://doi.org/10.32387/ prokla.v48i190.28

Raphael, Lutz (2019: Jenseits von Koble und Stabl. Eine Gesellschaftsgeschichte Westeuropas nach dem Boom. Frankfurt/M.

Reckwitz, Andreas (2017): Die Gesellschaft der Singularitäten. Zum Strukturwandel der Moderne. Frankfurt/M.

Rehage, Ruben (2018): Warum der Untergang der SPD nicht aufzuhalten ist. URL: https://www.stern.de/politik/deutschland/ spd--warum-der-untergang-der-sozialdemokraten-nicht-aufzuhalten-ist-8414982. html, Zugriff: 27.6.2019.

Ritsert, Jürgen (1998) Soziale Klassen. Münster.

Rhodes, Martin (2015): Labour Markets, welfare states, and the dilemmas of European social democracy. In: Keating, Michael/ McCrone, David (Hg.: The crisis of social democracy in Europe. Edinburgh: 140-155.

Sablowski, Thomas/Thien, Günter (2018): Die Arbeiterklasse und die Linke - kein Problem? In: PROKLA 48(190): 55-72 https:// doi.org/10.32387/prokla.v48i190.32

Seibert, Thomas (2016): Die Arbeiterklasse wird's nicht tun. URL: https://www.neuesdeutschland.de/artikel/1034515.rot-rotgruen-die-arbeiterklasse-wird-s-nicht-tun. html, Zugriff: 4.7.2019. 
Spier, Tim (2011): Wie aktiv sind die Mitglieder von Parteien. In: von Alemann, Ulrich (Hg.): Parteimitglieder in Deutschland. Wiesbaden: 97-119. https://doi. org/10.1007/978-3-531-92900-2_7

Stegemann, Bernd (2018): Die Moralfalle. Für eine Befreiung linker Politik. Berlin.

Thien, Günter (2018): Die verlorene Klasse. ArbeiterInnen in Deutschland. Münster.

Vester, Michael (2019): Von Marx bis Bourdieu. Klassentheorie als Theorie der Praxis. In: Klassen - Fraktion - Milieus. Beträge zur Klassenanalyse (1). Rosa Luxemburg Stiftung. Manuskripte 22. Berlin: 9-67.

von Beyme, Klaus (2000a): Parteien im Wandel. Von den Volksparteien zu den professionalisierten Wählerparteien. Wiesbaden. https:// doi.org/10.1007/978-3-322-90730-1
Ders. (2000b): Funktionenwandel der Parteien in der Entwicklung von der Massenmitgliederpartei zur Partei der Berufspolitiker. In: Niedermayer, Oskar u.a. (Hg.): Parteiendemokratie in Deutschland. Wiesbaden: 315-340.

Walter, Franz (2010): Vom Milieu zum Parteienstaat. Lebenswelten, Leitfiguren und Politik im historischen Wandel. Wiesbaden. https:// doi.org/10.1007/978-3-531-92213-3

Ders./Micus, Matthias (2011): Wieder zurück? Die SPD als Volkspartei. In: Zeitschrift für Staats- und Europawissenschaften 9(3): 383-409. https://www.doi. org/10.5771/1610-7780-2011-3-383

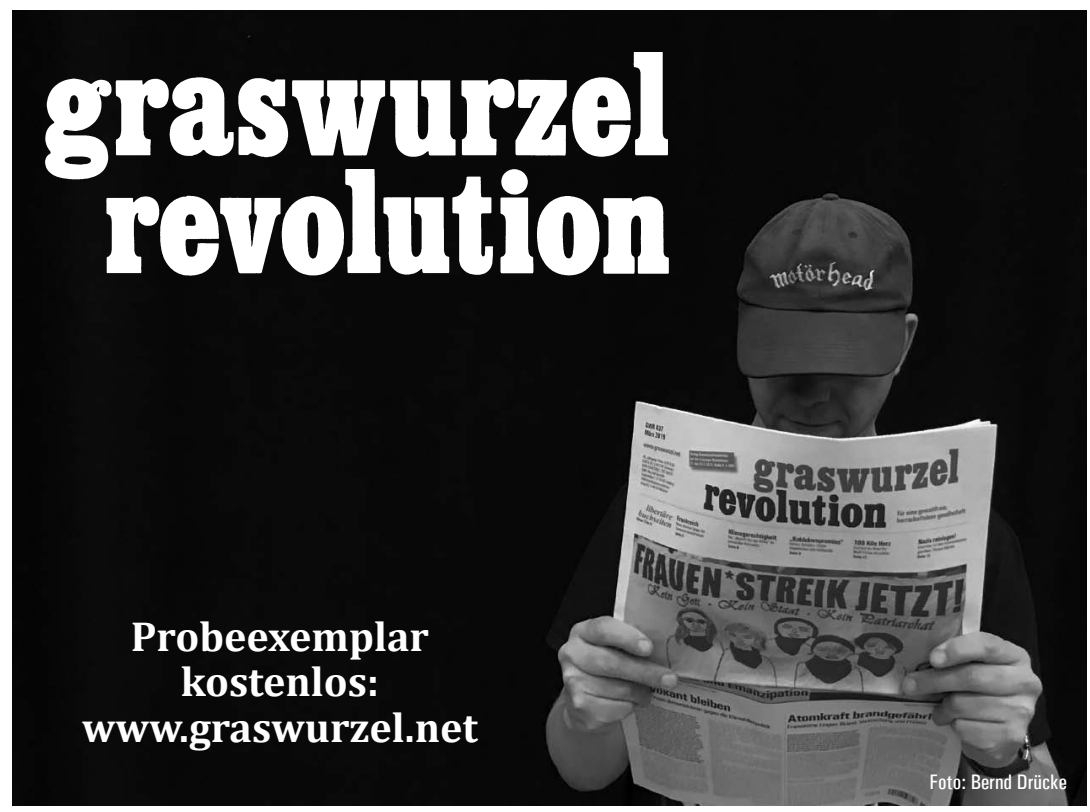

